\title{
Einleitung: Migration und (demokratische) politische Kultur - ein dynamisches und polarisierendes Thema?
}

\author{
Gert Pickel · Antje Röder · Andreas Blätte
}

Online publiziert: 5. Februar 2018

(C) Springer Fachmedien Wiesbaden GmbH, ein Teil von Springer Nature 2018

Seit 2015 haben sich die Koordinaten der politischen Welt in der wir leben verändert. Der im Rückblick fast schon beschaulich anmutende Stil der innenpolitischen Debatten wurde durch ein globales Ereignis aufgebrochen, welches von vielen deutlich markierend als „Flüchtlingskrise“ bezeichnet wurde. Schlagartig wuchsen die Migrationsbewegungen Richtung Europa an. Bilder von hoffnungslos überfüllten, kaum seetüchtigen Booten, von Flüchtlingslagern und Flüchtlingstrecks, von Menschen, die an Stacheldrahtzäunen rüttelten, und von Menschen, die in Deutschland und Europa ankamen und kaum geregelt versorgt werden konnten, nahmen über die Fernsehschirme Einzug in die Wohnzimmer. Nachrichten über Todesfälle im Mittelmeer einerseits, schwierige oder scheiternde politische Einigungen kamen hinzu. Diese Entwicklung hatte tiefgreifende Auseinandersetzungen im öffentlichen Raum zur Folge. Vielfältige und kontroverse mediale Diskurse, aber auch ein bis in die derzeitigen Verhandlungen über mögliche Koalitionen in der deutschen Politik nach den Bundestagswahlen 2017 hinreichender Streit über (die richtigen) politischen Entscheidungen prägen seitdem das Feld der Politik in Deutschland und Europa. Selbst der deutliche Rückgang der Zahl der Flüchtlinge seit 2016 hat die Debatten nicht zum Erliegen gebracht. Der Themenkomplex Flucht, Asyl und Migration überlagert nun schon seit geraumer Zeit alle anderen politischen Themen. Selbst die Intensität der Debattenlage von 1991-1993, als das gerade erst vereinte Deutschland einen historischen Höchststand der Asylbewerberzahlen politisch kontrovers verarbeitete (Herbert 2001), relativiert sich angesichts der heutigen Situation. Zudem handelt es sich um (mindestens) eine europaweite Diskussion und Problemlage,

Prof. Dr. G. Pickel ( $\bowtie)$

Theologische Fakultät, Abteilung Religions- und Kirchensoziologie, Professur für Religions- und Kirchensoziologie, Universität Leipzig, Martin-Luther-Ring 3, 04109 Leipzig, Deutschland

E-Mail: pickel@rz.uni-leipzig.de 
was vergleichende Betrachtungsweisen und Erklärungsansätze dringend geboten erscheinen lässt.

Ein Ende der Präsenz des Migrationsthemas scheint nicht in Sicht. Dabei sind bei genauer Sicht, - und dies zeigen auch einige der in diesem Special Issue der Zeitschrift für Vergleichende Politikwissenschaft vorliegenden Beiträge -, die Grundlagen dieser nun in heftiger Form aufbrechenden Konflikte sowohl in Politik als auch in der Bevölkerung schon länger latent vorhanden (Bauman 2016; Sniderman und Hagendoorn 2007). Allein schien es so zu sein, als würden die kontinuierlichen Migrationsprozesse der letzten Jahrzehnte politisch hinreichend kanalisiert sein und in einem globalisierten, individualisierten und transnational aufgestellten Europa höchstens noch ein zu bewältigendes logistisches bzw. politisch-administratives Problem darstellen (Baringhorst et al. 2006; Brettell und Hollifield 2000). Dabei hätten einem frühe Bücher und Bucherfolge zu denken geben können. Hierfür stehen, als umstrittene Schriften, der mittlerweile klassische „Clash of Civilizations“ von Samuel Huntington (1996), „Exodus. How Migration is Changing our World“ von Paul Collier (2013), sowie - als eine nicht immer deutlich wahrgenommene Zäsur der Debatte in Deutschland - das mit über 1,5 Millionen verkauften Exemplaren in der Geschichte der Bundesrepublik meistverkaufte Sachbuch „Deutschland schafft sich ab" von Thilo Sarrazin (2010). Am Erfolg des Sarrazin-Buchs wie an immer wieder neu belebbaren Leitkultur-Debatten lässt sich ablesen, dass deutlich schwieriger politisch zu befriedende kulturelle und identitäre Argumente gegenüber pragmatischen politischen Lösungsproblemen (hinsichtlich Arbeitsplätzen und Inklusion auf dem Arbeitsmarkt) die Oberhand gewonnen haben. Diese Diskussion hat sich mittlerweile, so etwa im Vergleich zur Zuwanderungsdebatte ab dem Frühjahr 2000, weniger auf die Umsetzung von Integration als eine zentrale Aufgabe der Gesellschaft gerichtet, die gelöst werden muss, als vielmehr auf die Frage danach, ob die Mehrheit der Bürger diese Integration überhaupt will. Die Argumente hier reichen von der Annahme einer generell mangelnden Integrationsfähigkeit von Immigranten, dem „Problem“ maßgeblich muslimischer Immigration in das demokratische, moderne, christliche und/oder säkulare Europa (zu dem die muslimischen Migranten kulturell nicht passen), bis hin zu der Blockadehaltung von bürgerlichen Mehrheiten und offener Ausländer- und Islamfeindlichkeit in der Folge der begleitenden Ausbreitung rechtspopulistischer Gruppierungen und Parteien.

Letztere haben sich das Migrationsthema und die Abwehr gegen Migration sehr erfolgreich als Leitthema zu eigen gemacht, wie ihre Wahlerfolge in verschiedenen europäischen Ländern in den letzten Jahren eindrucksvoll demonstrieren. Man kann vermutlich sogar mit Recht behaupten, dass diese Erfolge ohne die Bezugnahme auf das Thema Migration und der daraus abgeleiteten Drohung einer ,kulturellen Überfremdung" so gar nicht möglich gewesen wären (siehe auch Bettina Westle und Eva-Maria Raschke). So ist es die Gruppe der Migranten, welche sowohl eine klare politische Agenda dieser Parteien zu entwickeln geholfen hat, wie sie auch Potential für Mobilisierung in den Bevölkerungen für rechtspopulistische Positionen mit sich brachte. Wie wichtig das Thema Religion in diesem Zusammenhang ist, zeigen die entsprechenden Beiträge in diesem Heft, mit Bezug auf die Regulierung von Religionsunterricht (Eva-Maria Euchner), Kontroversen um religiöse Rechte (Sarah Carol), Zuschreibungen von Fremdheit und Bedrohlichkeit zur sozialen Gruppe 
„der Muslime“ (Gert und Susanne Pickel) oder die entsprechend geprägte Medienberichterstattung (Tobias Müller). Und fast immer geht es in diesen Debatten um die Rolle des Islam, was insbesondere in den Beiträgen von Sarah Carol sowie von Holger Kolb in Hinblick auf Religionspolitik in Deutschland hinterfragt wird. „Der Islam“ und „die Muslime“ eignen sich, aufgrund ihrer kulturellen Fremdheit sowie einer leichten Zuschreibung als gefährlich, besonders gut für die Reflexion eigener nationalistischer wie identitätsbezogener Wünsche und Hoffnungen.

In Folge dieser gesellschaftlichen und politischen Entwicklungen hat auch die Zahl der Studien zu Migration in den letzten Jahren merklich zugenommen. Der Umgang von Politikern mit dem Thema Migration, bewusste Veränderungen des Sprachgebrauchs (z.B. von „Flüchtlingen“ zu „Geflüchteten“) wie auch die Einstellungen gegenüber Einwanderern, Flüchtlingen wie auch Muslimen bzw. die in den Zuwanderungsgruppen vorherrschenden Einstellungen und Erwartungen selbst werden erforscht. Die frühere Dominanz der primär „mehrheitsgesellschaftlichen“ Perspektive ist mittlerweile zumeist überwunden. Große Teile dieses Spektrums werden Leserinnen und Leser auch in diesem Special Issue wiederfinden. Gleichwohl bleibt einiges an Forschungsbedarf offen. Zum einen hinkt die Forschung zu Migrantengemeinschaften den Entwicklungen anhaltend hinterher. Die empirische Erforschung von Identitätsbildungsprozessen und der Einfluss der sozialen Kontexte auf diese steht noch immer Anfang. Die neuen Zu- bzw. Einwanderer bieten der Forschung reichlich Stoff und Anlass zur theoretischen Fortentwicklung. Neue Perspektiven, wie jene der ,,postmigrantischen Gesellschaft“ (Foroutan et al. 2014) fordern genauso dazu auf, die Kategorien und den Fokus der Forschung zu überdenken, wie Diskussionen im Feld der Extremismusforschung. Auch die Mechanismen, warum in der Mehrheitsbevölkerung migrationsfreundliche oder eher migrationsablehnende Einstellungen und Verhaltensweisen Ausbreitung finden, sind bislang nicht zufriedenstellend entschlüsselt, wenngleich dies noch eines der am besten erforschten Teilbereiche mit langer Tradition ist (siehe z. B. Blumer 1958). Nicht viel anders sieht es mit der vergleichenden Perspektive aus. Die Literatur der Citizenship Studies verfolgt zwar oft eine makro-komparative Perspektive, doch ansonsten sind die Betrachtungen auf einzelne Länder zentriert, über diese Einzelfälle hinausreichende Erklärungsmodelle sind Mangelware. Dabei ist es vor allem die kulturelle Seite der Migration und ihrer Folgen in den (politischen) Kulturen der Immigrationsländer, welche in ihren Voraussetzungen und Wirkungen noch nicht ausgedeutet ist. Einen Beitrag zu dieser Debatte liefern in diesem Band Polly Diven und Stefan Immerfall mit dem Vergleich deutscher und US amerikanische Reaktionen auf die Flüchtlingskrise, sowie Sybille Münch im Vergleich von Integrationsdiskursen in Deutschland und Großbritannien, während Sascha Kranich und Uwe Hunger fünf Länder in Bezug auf ihre Einwanderungspolitik vergleichen. Aber auch der innerdeutsche Vergleich kann hierbei gewinnbringend sein, wie die Beiträge von Daniel Kubiak, Rene Böhme sowie von Birgit Glorius und Anne Schondelmayer aufzeigen. Dass diese Debatten nicht komplett neu sind, zeigt der Beitrag von Felix Krawatzek und Gwendolyn Sasse in ihrer historischen Analyse deutscher Migranten in den Vereinigten Staaten.

Bemerkenswert ist die Beobachtung, dass es bei den heute interessierenden Fragestellungen oft eher auch um Integration oder Inklusion, aber weniger um konkrete 
Prozesse der Migration selbst geht. So scheint es manchmal, als würde sich eine eigenständige Integrationsforschung in Differenz oder Ergänzung zu transnationalen Linien der Migrationsforschung etablieren, auch wenn beide Linien einander stark verbunden bleiben (Portes 1997). Diese Verschiebung findet sich nicht nur mit Blick auf die stärkere Betonung von Arbeiten der Umfrageforschung, sondern auch in Arbeiten, welche den Schwerpunkt ihrer Analyse im regionalen Bereich anlegen (Charlotte Räuchle). Integration oder gar der weiter reichende Begriff der Inklusion prägen dabei nicht nur politische Debatten, sondern können auch als Begriffe wahrgenommen werden, an denen sich die (sich teils polarisiert gegenüberstehenden) Gruppen mit unterschiedlicher Haltung zur Migrationspolitik identifizieren lassen. Diese Ausdifferenzierung ist durchaus nachvollziehbar, ist doch wahrscheinlich der am stärksten durchschlagende gesellschaftliche Effekt der derzeitigen Migrationsprozesse nach Europa oder in den USA, ihre Auswirkung auf die Veränderung der dortigen Gesellschaften, deren Struktur und Kultur. ${ }^{1}$

Mit diesen Überlegungen ist man bereits recht nahe beim Begriff und Phänomen der politischen Kultur (Almond und Verba 1963; Easton 1975; Lipset 1981; zusammenfassend Pickel und Pickel 2006). Im öffentlichen Blick wird diese zwar selten im Zusammenhang mit Migration und Integration explizit angesprochen, aber implizit ist die Beziehung von Migration und Immigration zu politischer Kultur, zum Zusammenhalt der Gesellschaft wie auch zum langfristigen Bestand der Demokratie sehr gut erkennbar und naheliegend. Gerade die Konjunktur des Begriffs „gesellschaftlicher Zusammenhalt“ ist ein Indiz für die aufkeimende Sorge, die sich aus den Integrationsprozessen und deren Gegenreaktionen ergibt. Dieser wirft die alte Frage neu auf, wann Integration als gelungen erachtet werden kann. Ist dies der Fall, wenn es über vielfältige Anpassungsprozesse aller Seiten zu einer Inklusion aller Mitglieder einer Gemeinschaft kommt? Doch wie viel an Gemeinschaft benötigt man? So wie eine vollständige Homogenität moderner (und plural aufgestellter) Gesellschaften weder realistisch noch wünschenswert ist, ohne einen gemeinsamen Grundkonsens und auf Basis maximaler Pluralität scheint der langfristige Erhalt einer Gemeinschaftsstruktur auch nicht möglich. Die Politikwissenschaft kann ihre alte Tradition des Pluralismus in Erinnerung rufen und darauf hinweisen, dass kulturelle und soziale Verhaltensweisen in modernen pluralistischen Gesellschaften eben plural sind. Gleichzeitig reicht dies für den Erhalt einer politischen Gemeinschaft auf Dauer mit Blick auf die politische Kulturforschung nicht aus. Hier bedarf es einer Legitimität bestimmter Grundlagen der politischen Gemeinschaft (Lipset 1981). Eine ist die Anerkennung einer demokratischen Ordnung, eine andere die Toleranz von Pluralität seitens aller Mitglieder einer Gemeinschaft, solange das Verhalten anderer nicht den Raum individueller Freiheiten maßgeblich einschränkt und zur Erosion der politischen Gemeinschaft führt. Auf der strukturellen Ebene hat ein bestimmtes Verständnis der demokratischen politischen Kultur in der Verfassung

\footnotetext{
1 Wobei auch nicht die Auswirkungen der Migration auf die Herkunftsstaaten der Migration übersehen werden dürfen. Die Auswirkungen von Migrationsbewegungen, deren sozialen und politischen Gründe und deren Folgen für diese Staaten und Regionen stellen ein weiteres eigenständiges Forschungsfeld von hoher Bedeutung dar. Speziell, wenn man dieses unter den Rahmenbedingungen von Globalisierung und transnationaler Migrationsbewegungen betrachtet.
} 
ihre Verstätigung gefunden. Gleichwohl geht es um deren Anerkennung - seitens der neu Hinzukommenden und der bereits Anwesenden. Die Beiträge von Sophia Hunger und von Eva Raschke und Bettina Westle betrachten dies aus Sicht der Migranten und deren politische Teilhabe, während der Beitrag von Verana Hambauer und Anja Mays sich mit den Einstellungen der bereits autochonen Bürger beschäftigen. Die aktuellen Debatten bestätigen in diesem Zusammenhang, dass gerade dem Wechselspiel von Integration und politischer Kultur eine große Bedeutung für das Funktionieren moderner Demokratien zukommt und in Zukunft zukommen wird.

Aber gerade dieses Verhältnis ist derzeit prekär. So wie etliche europäische, speziell osteuropäische, Länder sich einer Auseinandersetzung mit Migration dadurch entzogen, einfach keine mehr ins Land zu lassen, dafür Sanktionen der Europäischen Union in Kauf nahmen und sogar deren Spaltung riskieren (siehe hier insbesondere auch den Beitrag von Paula Beger), so scheint sich in den Bevölkerungen Europas eine nicht unwesentliche Abneigungshaltung gegenüber Migranten zu etablieren (Gert und Susanne Pickel). Nicht nur diese Beobachtung erfordert fast zwangsläufig eine komparative Perspektive auf die derzeit stattfindenden Phänomene. Genau genommen handelt es sich bei der Referenzgruppe für die Ablehnung und Zuschreibung gruppenbezogener Vorurteile meist um muslimischen Migranten (Pickel und Yendell 2016). Dabei wird, wie Behrouz Alikhani und Inken Rommel in ihrem Beitrag argumentieren, häufig über ,kulturelle Unterschiede“ geredet und so eine Abgrenzung vollzogen. Die Auseinandersetzungen haben sich dabei auf der Ebene der politischen Kultur - also in den Einstellungen der Bürger, bzw. verschiedener Gruppen von ihnen - manifestiert, so dass man mittlerweile sogar eine grundsätzliche Differenzierung der Gesellschaften in Kosmopoliten und Kommunitaristen diskutiert (Calhoun 2002, 2007; Merkel 2017). Erstere haben sich mit den schnellläufigen, durch Globalisierung beschleunigten Veränderungen der Umwelt gut angepasst und profitieren davon, während letztere immer stärk in eine Abwehrhaltung gegenüber diesen Veränderungen der Moderne entwickeln. Es handelt sich eben hierbei auch um Wertefragen, und bei diesen ist Veränderung eher langsam, sowohl bei der Mehrheitsbevölkerung als auch bei Migranten (Röder und Mühlau 2014). Skeptiker meinen sogar, sie wären gar nicht wirklich verhandelbar (Huntington 1996). Der Streit über Migration und Integration anderer sozialer Gruppen ist dabei der Angelpunkt, an dem sich diese Wertunterschiede treffen. Es ist auch der Punkt, an dem kollektive Identitäten und (oft fehlende) Intergruppenkontakte als Erklärungsmuster für Auseinandersetzungen über Migration und Migranten ins Spiel kommen (Pettigrew 1998; Tajfel 1982). Dies betrifft sowohl ihre Anwesenheit, ihre Integration als auch die Gewährleistung von Staatsbürgerrechten (Koopmans et al. 2005). Und dieser Streit findet sich wieder in der politischen Kultur, stellt sie sich doch seit ihrer Genese aus dem, (Integration in den Vordergrund rückenden) Strukturfunktionalismus die Frage, wie politische Systeme überleben können. Ihre Antwort ist dabei immer der Verweis auf ihre Legitimität in der Bevölkerung und so etwas wie ein Set an gemeinsamen Wertvorstellungen, die alle Mitglieder der Gesellschaft teilen. Dieser Grundkonsens und die politische Unterstützung des demokratischen Systems scheinen aber unter den Rahmenbedingungen neuer Integrationsherausforderungen und der Diskussionen über sie an einigen Stellen ins Wanken gekommen zu sein. 
Es bestehen also hinreichende Fragen zum Verhältnis von Migration, Integration und politischer Kultur. Dies war der Ausgangspunkt für das Ihnen nun vorliegende Special Issues der Zeitschrift für Vergleichende Politikwissenschaft. Einen komparativen Blick aufzunehmen, der die Bereiche Migration, Integration und politische Kultur aus unterschiedlichen Perspektiven beleuchtet und ihre Verbindungen aufzeigt war das Ziel. Der Zuspruch zum Thema war überwältigend. In einem Workshop Sommer 2016 nahmen über 40 Personen teil und über 30 Einreichungen wurden in den Peer Review Prozess eingespeist. Es liegt in der Natur dieses Prozesses, dass nicht alle Beiträge den Weg ins Heft gefunden. Wir danken allen, die sich in diesen Prozess eingebracht haben, den Autorinnen und Autoren, und besonders den Gutachterinnen und Gutachtern. Der trotzdem noch beachtliche Umfang des vorliegenden Special Issues zeigt allerdings nur zu deutlich die Brisanz des Themas - und das Interesse daran. Was deutlich wird ist, dass die religiöse Identität genauso eine Rolle für den Erfolg von Integration spielt, wie eine genaue Kontextbeobachtung zur Einschätzung von Wirkungsmechanismen notwendig ist. Eine Auseinandersetzung mit politischer Kultur und Migration setzt zudem voraus, dass man die Brücken zwischen Migration, Integration und politischer Kultur aus historischer Perspektive und interdisziplinärer beleuchtet. Auswirkungen auf das Wahlverhalten sind genauso beobachtbar, wie regionale Anstrengungen, die auf politischer Ebene unternommen werden müssen. Dabei wird auch ein noch erheblicher Bedarf an empirischen Studien deutlich. Und dies nicht nur aus rein wissenschaftlichem Interesse. Es ist derzeit kein anderes Thema erkennbar, an dem seitens der handelnden Politik so viel Interesse besteht, als an der Brückenbeziehung zwischen Migration, Integration und politischer Kultur. Wenn das vorliegende Special Issue zumindest etwas zur Beantwortung der relevanten Fragen als auch zu ihrer Präzisierung beiträgt, so hat es seinen Zweck erreicht.

Danksagung Wir danken an dieser Stelle den Herausgebern der Zeitschrift für Vergleichende Politikwissenschaft für die Möglichkeit das vorliegende Special Issue umzusetzen.

\section{Literatur}

Almond, Gabriel, und Sidney Verba. 1963. The civic culture. Political attitudes and democracy in five nations. Princeton: Princeton University Press.

Baringhorst, Sigrid, James F. Hollifield, und Uwe Hunger (Hrsg.). 2006. Herausforderung Migration Perspektiven der vergleichenden Politikwissenschaft. Berlin: LIT.

Bauman, Zygmunt. 2016. Die Angst vor den anderen. Ein Essay über Migration und Panikmache. Berlin: Suhrkamp.

Blumer, Herbert. 1958. Race prejudice as a sense of group position. Pacific Sociological Review 1:3-7.

Brettell, Caroline B., und James F. Hollifield. 2000. Migration theory. Talking across disciplines. London: Routledge.

Calhoun, Craig. 2002. Imagining solidarity: cosmopolitanism, constitutional patriotism, and the public sphere. Political Culture 14(1):141-171.

Calhoun, Craig. 2007. Nations matter. Culture, history and the cosmopolitan dream. Abingdon: Routledge. Collier, Paul. 2013. Exodus. How migration is shaping our world. Oxford: Oxford University Press.

Easton, David. 1975. A re-assessment of the concept of political support. British Journal of Political Science 5:435-457.

Foroutan, Naika, Coskun Canan, Sina Arnold, Benjamin Schwarze, Steffen Beigang, und Dorina Kalkum. 2014. Deutschland postmigrantisch I. Gesellschaft, Religion, Identität. Erste Ergebnisse. Berlin: BIM. 
Herbert, Ulrich. 2001. Geschichte der Ausländerpolitik in Deutschland. Saisonarbeiter, Gastarbeiter, Zwangsarbeiter. München: Beck.

Huntington, Samuel P. 1996. Kampf der Kulturen. Die Neugestaltung der Weltpolitik im 21. Jahrhundert. Wien: Europa Verlag.

Koopmans, Ruud, Paul Staham, Marco Giugni, und Florence Passy. 2005. Contested citizenship. Immigration and cultural diversity in Europe. Minneapolis: University of Minnesota Press.

Lipset, Seymour. 1981. Political man: the social bases of politics. Baltimore: Penguin.

Merkel, Wolfgang. 2017. Kosmopolitismus versus Kommunitarismus: Ein neuer Konflikt in der Demokratie. In Parties, governments and elites. The comparative study of democracy, Hrsg. Phillip Harfst, Ina Kubbe, und Thomas Poguntke, 9-23. Wiesbaden: Springer.

Pettigrew, Thomas F. 1998. Intergroup contact theory. Annual Review of Psychology 49:65-85.

Pickel, Gert, und Alexander Yendell. 2016. Islam als Bedrohung? Beschreibung und Erklärung von Einstellungen zum Islam im Ländervergleich. Zeitschrift für Vergleichende Politikwissenschaft 10:273-309.

Pickel, Susanne, und Gert Pickel. 2006. Politische Kultur- und Demokratieforschung. Eine Einführung. Wiesbaden: VS.

Portes, Alejandro. 1997. Immigration theory for a new century: some problems and opportunities. International Migration Review 31:799-825.

Röder Antje, und Peter Mühlau. 2014. Are they acculturating? Europe's immigrants and gender egalitarianism. Social Forces 92(3):899-928.

Sarrazin, Thilo. 2010. Deutschland schafft sich ab. München: DVA.

Sniderman, Paul M., und Louk Hagendoorn. 2007. When ways of life collide. Princeton: Princeton University Press.

Tajfel, Henri. 1982. Social identity and intergroup relations. Cambridge: University Press. 